Appl Math Optim 55:145-161 (2007)

DOI: $10.1007 / \mathrm{s} 00245-006-0883-0$

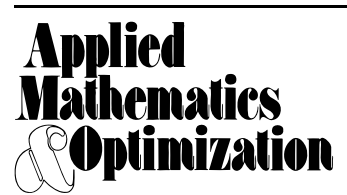

(C) 2007 Springer Science+Business Media, Inc.

\title{
Generalized Harmonic Functions and the Dewetting of Thin Films*
}

\author{
Giles Auchmuty ${ }^{1}$ and Petr Klouček ${ }^{2}$ \\ ${ }^{1}$ Division of Mathematical Sciences, National Science Foundation, \\ Arlington, VA 22230, USA \\ and \\ Department of Mathematics, University of Houston, \\ 4800 Calhoun, TX 77204-3008, USA \\ gauchmut@nsf.gov \\ ${ }^{2} \mathrm{TLC}^{2}$, University of Houston, \\ 4800 Calhoun, TX 77204, USA \\ and \\ Institut de Mathèmatiques, Universitè de Neuchâtel, \\ Rue Emile Argand 11, CH-2007 Neuchâtel, Switzerland \\ kloucek@mac.com
}

\begin{abstract}
This paper describes the solvability of Dirichlet problems for Laplace's equation when the boundary data is not smooth enough for the existence of a weak solution in $H^{1}(\Omega)$. Scales of spaces of harmonic functions and of boundary traces are defined and the solutions are characterized as limits of classical harmonic functions in special norms. The generalized harmonic functions, and their norms, are defined using series expansions involving harmonic Steklov eigenfunctions on the domain. It is shown that the usual trace operator has a continuous extension to an isometric isomorphism of specific spaces. This provides a characterization of the generalized solutions of harmonic Dirichlet problems. Numerical simulations of a model problem are described. This problem is related to the dewetting of thin films and the associated phenomenology is described.
\end{abstract}

Key Words. Steklov eigenvalue problem, Trace spaces, Generalized harmonic functions, Dewetting.

AMS Classification. 35P05, 35J05, 35J55, 46F99, 65N25, 76A20.

* The second author was supported in part by Grants NSF ACI-0325081 and NSF CCR-0306503 and by the European Commission via MEXC-CT-2005-023843. 


\section{Introduction}

This paper will describe "very weak" or "generalized" solutions of Laplace's equation when the boundary data is not sufficiently nice for the problem to have a weak solution in $H^{1}(\Omega)$. We shall show that such solutions may be described spectrally via expansions in terms of harmonic Steklov eigenfunctions of the region. Moreover, these spectral approximations can be used to obtain good numerical simulations of the solutions. A model problem with discontinuous boundary data related to the dewetting of thin films is treated explicitly and the solution is interpreted physically.

To describe these results, the essential properties of harmonic Steklov eigenproblems are summarized first. They are based on results in [1] and [2]. Then, in Section 3, a scale of spaces of generalized harmonic functions is defined which includes the class of finiteenergy harmonic functions. In Section 4 certain trace spaces are defined as was done in [2]. These definitions are intrinsic and apply to more general domains than the usual theory of trace spaces. With these definitions, the boundary trace operator is shown to be an isometric isomorphism between certain classes of (generalized) harmonic functions on $\Omega$ and specific spaces of functions on the boundary $\partial \Omega$. This result may also be viewed as a description of the unique solvability of a Dirichlet problem for the Laplacian on $\Omega$ and this is described in Section 5. An efficient computational implementation of the Steklov spectral approach for the classical Dirichlet problem for Laplace's equation is described in [7].

For the unit disc in the plane, the Steklov eigenvalues and eigenfunctions are known explicitly and a model problem with discontinuous boundary data is solved explicitly in Section 6. The solution is a bounded function that is not of class $H^{1}$. Numerical simulations and approximations are described. The behavior of these solutions as a model for the dewetting of thin films is discussed in Section 7.

\section{Harmonic Functions and Steklov Eigenproblems}

In this section some basic assumptions and definitions are provided for use in this paper. In particular the subspace $\mathcal{H}(\Omega)$ of all weakly harmonic functions in $H^{1}(\Omega)$ will be defined and a natural orthonormal basis of this space is described.

Let $\Omega$ be a non-empty, connected bounded open subset of $\mathbb{R}^{n}$ with boundary $\partial \Omega:=$ $\bar{\Omega} \backslash \Omega$ satisfying

(B1) $\partial \Omega$ is a finite union of connected, closed, Lipschitz surfaces, and each component of $\partial \Omega$ has finite surface area.

Let $H^{1}(\Omega)$, resp. $H_{0}^{1}(\Omega)$, be the usual real spaces of the Sobolev function on $\Omega$. The standard inner product on $H^{1}(\Omega)$ is defined by

$$
\langle u, v\rangle_{H^{1}}:=\int_{\Omega}[\nabla u(x) \cdot \nabla v(x)+u(x) v(x)] .
$$

All functions, and function spaces, in this paper are assumed to be real valued. (B1) is sufficient to ensure that Rellich's theorem that the embedding $H^{1}(\Omega) \mapsto L^{2}(\Omega)$ is compact holds; see [6]. Let $d \sigma$ represent the Hausdorff $(n-1)$-dimensional measure on 
$\partial \Omega$. Then the Lebesgue space $L^{2}(\partial \Omega, d \sigma)$ is a real Hilbert space under the inner product

$$
\langle u, v\rangle_{\sigma}:=\frac{1}{\sigma(\partial \Omega)} \int_{\partial \Omega} u v d \sigma .
$$

When (B1) holds, then the trace operator $\Gamma: H^{1}(\Omega) \mapsto L^{2}(\partial \Omega, d \sigma)$ will be compact; see Section 4.3 of [5]. In fact the analysis of this paper will hold under the following (weaker) assumption:

(B2) Rellich's theorem and the compact trace theorem hold for $H^{1}(\Omega)$.

Another inner product on $H^{1}(\Omega)$ is defined by

$$
[u, v]_{\partial}:=\int_{\Omega} \nabla u(x) \cdot \nabla v(x) d x+\langle u, v\rangle_{\sigma} .
$$

This is an equivalent inner product on $H^{1}(\Omega)$ when (B1) or (B2) holds; see Corollary 6.2 of [1] or Theorem 21A of [11].

\subsection{The Space $\mathcal{H}(\Omega)$ of Weakly Harmonic Functions}

A function $u \in H^{1}(\Omega)$ is said to be weakly harmonic on $\Omega$ provided it satisfies the usual weak form of Laplace's equation, namely

$$
\int_{\Omega} \nabla u(x) \cdot \nabla \varphi(x) d x=0 \quad \text { for all } \quad \varphi \in C_{\mathrm{C}}^{\infty}(\Omega) .
$$

Here $C_{\mathrm{C}}^{\infty}(\Omega)$ denotes the space of all infinitely differentiable functions with compact support in $\Omega$. The subspace of $H^{1}(\Omega)$ of all functions that satisfy (2.4) is denoted by $\mathcal{H}(\Omega)$ and is a closed subspace of $H^{1}(\Omega)$. Sometimes it is called the space of finite-energy harmonic functions on $\Omega$.

This definition and the inner product (2.3) yield that

$$
H^{1}(\Omega)=H_{0}^{1}(\Omega) \oplus_{\partial} \mathcal{H}(\Omega),
$$

as $H_{0}^{1}(\Omega)$ is the closure of $C_{\mathrm{C}}^{\infty}(\Omega)$ in $H^{1}(\Omega)$. Here $\oplus_{\partial}$ indicates an orthogonal sum with respect to the inner product (2.3). That is, a function $u \in H^{1}(\Omega)$ is weakly harmonic if and only if it is $\partial$-orthogonal to $H_{0}^{1}(\Omega)$.

\subsection{Steklov Eigenvalues and Steklov Eigenfunctions}

A non-trivial function $s \in H^{1}(\Omega)$ is said to be a harmonic Steklov eigenfunction on $\Omega$ corresponding to the Steklov eigenvalue $\delta$ if it satisfies

$$
\int_{\Omega} \nabla s(x) \cdot \nabla \varphi(x) d x=\delta\langle s, \varphi\rangle_{\sigma} \quad \text { for all } \quad \varphi \in H^{1}(\Omega) .
$$

Taking $\varphi \in C_{\mathrm{C}}^{\infty}(\Omega)$ one sees that any such eigenfunction is weakly harmonic on $\Omega$. Consequently it is in $\mathcal{H}(\Omega)$. This equation (2.6) is the usual weak form of the boundary value problem

$$
\Delta s=0 \quad \text { on } \Omega \text { with } \quad \nabla s \cdot v=\frac{\delta}{\sigma(\partial \Omega)} s \quad \text { on } \partial \Omega .
$$


The least eigenvalue of the Steklov eigenproblem (2.6) is $\delta_{0}=0$ corresponding to the eigenfunction $s_{0} \equiv 1$. This eigenvalue is simple when $\Omega$ is connected. Successive Steklov eigenvalues and the associated eigenfunctions may be described recursively using variational principles; see Sections 6 and 7 of [1]. An efficient computational approach to obtaining the Steklov eigenpairs via a generalized eigenvalue formulation is developed in [7]. Let the Steklov eigenvalues be

$$
0=\delta_{0}<\delta_{1} \leq \delta_{2} \leq \cdots \leq \delta_{k} \leq \cdots
$$

and let $\mathcal{S}:=\left\{s_{k} \mid k \geq 0\right\}$ be a corresponding family of eigenfunctions. They may be chosen to be orthonormal with respect to the $\partial$-inner product (2.3) so that

$$
\left[s_{j}, s_{k}\right]_{\partial}= \begin{cases}1, & \text { if } j=k, \\ 0, & \text { otherwise }\end{cases}
$$

In Theorems 7.2 and 7.3 of [1] it is proven that $\delta_{k} \rightarrow+\infty$ as $k \rightarrow+\infty$ and that $\mathcal{S}$ is an orthonormal basis in $\mathcal{H}(\Omega)$.

When $u \in \mathcal{H}(\Omega)$, then

$$
u(x):=\sum_{k=0}^{\infty}\left[u, s_{k}\right]_{\partial} s_{k}(x)
$$

will be called the Steklov expansion of $u$ with respect to $\mathcal{S}$ and the $K$ th Steklov approximation of $u$ will be the finite sum

$$
u_{K}(x):=\sum_{k=0}^{K}\left[u, s_{k}\right]_{\partial} s_{k}(x) .
$$

The following theorem summarizes some important properties of these Steklov approximations and depends on standard results from Hilbert space theory.

Theorem 2.1. Let $\mathcal{S}$ be a maximal d-orthonormal family of harmonic Steklov eigenfunctions on $\Omega$ as above. Suppose $u \in \mathcal{H}(\Omega)$, then $u_{K} \in C^{\infty}(\Omega) \cap \mathcal{H}(\Omega)$ for each $K \geq 1$. Moreover, $u_{K}$ converges to $u$ in $H^{1}(\Omega)$ as $K \rightarrow \infty$ and

$$
\|u\|_{\partial}^{2}=\sum_{k=0}^{\infty}\left[u, s_{k}\right]_{\partial}^{2} .
$$

Proof. Each Steklov eigenfunction is weakly harmonic on $\Omega$ and in $H^{1}(\Omega)$. Hence, it follows from Weyl's lemma that $s_{k} \in C^{\infty}(\Omega)$. Thus $u_{K} \in C^{\infty}(\Omega)$ for any $K \geq 1$. The strong convergence in $H^{1}(\Omega)$ follows from the Riesz-Fischer Theorem since $\mathcal{S}$ is a orthonormal basis of $\mathcal{H}(\Omega)$. Finally (2.9) follows from Parseval's equality.

\section{Hilbert Spaces of Generalized Harmonic Functions}

The space $\mathcal{H}(\Omega)$ defined in the preceding section is associated with the usual theory of weak solutions of harmonic boundary value problems on $\Omega$. Here we shall define some 
classes of generalized harmonic functions that are appropriate for solving harmonic Dirichlet problems when the boundary data is not in $H^{1 / 2}(\partial \Omega)$. These classes will be defined via their Steklov series expansions. As in the previous section, $\mathcal{S}$ denotes a maximal $\partial$-orthonormal family of harmonic Steklov eigenfunctions on $\Omega$.

Let $\mathcal{H}_{F}(\Omega)$ be the class of all finite linear combinations of functions in $\mathcal{S}$, then

$\mathcal{H}_{F}(\Omega) \subseteq C^{\infty}(\Omega) \cap H^{1}(\Omega)$.

Moreover, $\mathcal{H}(\Omega)$ is the closure of $\mathcal{H}_{F}(\Omega)$ with respect to the topology induced by the $\partial$-norm from Theorem 2.1 above.

Suppose $u=\sum_{j=0}^{\infty} c_{j} s_{j}(x)$ is in $\mathcal{H}_{F}(\Omega)$. For any real number $s \in \mathbb{R}$, define the s-norm of $u$ by

$$
\|u\|_{s}^{2}:=\sum_{j=0}^{\infty}\left(1+\delta_{j}\right)^{2(s-1)} c_{j}^{2} .
$$

Here $\delta_{j}$ is the $j$ th harmonic Steklov eigenvalue of the region $\Omega$ and this sum is finite as there are only finitely many nonzero $c_{j} \mathrm{~s}$.

Definition 3.1. For any $s \in \mathbb{R}$, define $\mathcal{H}^{s}(\Omega)$ to be the closure of the space $\mathcal{H}_{F}(\Omega)$ with respect to the s-norm (3.1).

For $s>1, \mathcal{H}^{s}(\Omega)$ is a dense subspace of the space $\mathcal{H}(\Omega)$ of weakly harmonic functions on $\Omega$. $\mathcal{H}^{s}(\Omega)$ may be regarded as the class of all Steklov expansions

$$
u:=\sum_{j=0}^{\infty} c_{j} s_{j}(x)
$$

for which the sum in (3.1) is finite. When $u, v$ are in $\mathcal{H}^{s}(\Omega)$ and have Steklov coefficients $\left\{c_{j}, d_{j}\right\}$, respectively, then

$$
\langle u, v\rangle_{s}:=\sum_{j=0}^{\infty}\left(1+\delta_{j}\right)^{2(s-1)} c_{j} d_{j}
$$

is an inner product on $\mathcal{H}^{s}(\Omega)$ and it will be a real Hilbert space under this inner product.

Note that with this definition $\mathcal{H}^{1}(\Omega)=\mathcal{H}(\Omega)$. When $s<1$ there will be generalized functions in $\mathcal{H}^{s}(\Omega)$ that are not $H^{1}$-functions on $\Omega$. Such functions may not be weakly harmonic in the sense of (2.4) but they are limits of classical $C^{\infty}$-harmonic functions in this s-norm. Hence they will be called generalized harmonic functions. These definitions define a scale of real Hilbert spaces with the following properties:

Theorem 3.2. Assume that the domain $\Omega$ satisfies either (B1) or (B2) and $s_{1}<s_{2}$, then

(i) $\mathcal{H}^{s_{2}}(\Omega)$ is dense in $\mathcal{H}^{s_{1}}(\Omega)$,

(ii) the embedding $\mathcal{H}^{s_{2}}(\Omega) \mapsto \mathcal{H}^{s_{1}}(\Omega)$ is compact,

(iii) if $s=(1-\theta) s_{1}+\theta s_{2}, \theta \in[0,1]$, the following interpolation inequality holds:

$$
\|u\|_{s} \leq\|u\|_{s_{1}}^{1-\theta}\|u\|_{s_{2}}^{\theta} \quad \text { for all } \quad u \in \mathcal{H}^{s_{2}}(\Omega) .
$$


Proof. This follows from standard arguments for such sequence spaces. See Section 5 of [2] for a detailed proof of a similar result.

A natural question is whether the functions in $\mathcal{H}^{s}(\Omega)$ are in the usual spaces $H^{s}(\Omega)$ for some definition of such spaces when $s \neq 1$ and the domain $\Omega$ has a sufficiently nice boundary.

\section{Boundary Traces of Generalized Harmonic Functions}

There is a well-known theory for the boundary traces of $H^{1}$-functions described in [9] and elsewhere. This theory requires considerable boundary regularity and is not very amenable to computation. A somewhat different description of trace spaces using Steklov eigenfunctions has recently been developed in [2]. This approach applies to somewhat more general domains, is intrinsic and involves norms that just depend on the quantities involved in Steklov expansions.

Let $\Gamma$ be the trace operator as in Section 2. When the Steklov eigenfunctions satisfy (2.7), then (2.6) yields

$$
\left\langle\Gamma s_{j}, \Gamma s_{k}\right\rangle_{\sigma}= \begin{cases}\left(1+\delta_{k}\right)^{-1}, & \text { if } j=k, \\ 0, & \text { otherwise. }\end{cases}
$$

This implies that the traces of the Steklov eigenfunctions on the boundary $\partial \Omega$ are orthogonal with respect to the standard $L^{2}$-inner product on $\partial \Omega$. For each $j \geq 0$, define

$$
g_{j}(x):=\sqrt{1+\delta_{j}} \Gamma s_{j}(x), \quad x \in \partial \Omega .
$$

Then $\mathcal{S}_{1}:=\left\{g_{j} \mid j \geq 0\right\}$ is an orthonormal set in $L^{2}(\partial \Omega, d \sigma)$ and Theorem 9.4 of [2] shows that $\mathcal{S}_{1}$ is maximal. That is $\mathcal{S}_{1}$ is an orthonormal basis of $L^{2}(\partial \Omega, d \sigma)$ and for any $f \in L^{2}(\partial \Omega, d \sigma)$ the partial sums

$$
f_{K}(x):=\sum_{m=0}^{K}\left\langle f, g_{m}\right\rangle_{\sigma} g_{m}(x)
$$

will be well-defined functions in $L^{2}(\partial \Omega, d \sigma)$ such that $f_{K} \rightarrow f$ as $K \rightarrow \infty$ in the $L^{2}$-norm.

For $s>0$, define the subspace $H^{s}(\partial \Omega)$ of $L^{2}(\partial \Omega, d \sigma)$ to be the space of all functions satisfying

$$
\|f\|_{s, \partial \Omega}^{2}:=\sum_{j=0}^{\infty}\left(1+\delta_{j}\right)^{2 s}\left\langle f, g_{j}\right\rangle_{\sigma}^{2}<\infty .
$$

Here the $\delta_{j}$ are harmonic Steklov eigenvalues on $\Omega$. The space $H^{s}(\partial \Omega)$ is a real Hilbert space under the inner product

$$
\langle f, h\rangle_{s, \partial \Omega}:=\sum_{j=0}^{\infty}\left(1+\delta_{j}\right)^{2 s}\left\langle f, g_{j}\right\rangle_{\sigma}\left\langle h, g_{j}\right\rangle_{\sigma} .
$$


When $s=0$ this yields $H^{0}(\partial \Omega)=L^{2}(\partial \Omega, d \sigma)$. Suppose $u \in \mathcal{H}_{F}(\Omega)$ has a Steklov expansion $u=\sum_{j=0}^{\infty} c_{j} s_{j}$. Apply $\Gamma$, then (4.2) yields

$$
\begin{aligned}
& \Gamma u(x)=\sum_{j=0}^{\infty} \frac{c_{j}}{\sqrt{1+\delta_{j}}} g_{j}(x) \text { and, from (4.4), } \\
& \|\Gamma u\|_{s, \partial \Omega}^{2}=\sum_{j=0}^{\infty}\left(1+\delta_{j}\right)^{2 s-1} c_{j}^{2} .
\end{aligned}
$$

When $u \in \mathcal{H}(\Omega)$, take $s=\frac{1}{2}$ then $\Gamma u \in H^{1 / 2}(\partial \Omega)$ from Theorem 2.1, so this definition agrees with the usual property of this trace space for the case of smooth $\partial \Omega$.

More details of this construction are provided in [2]. This definition may be extended to the case $s<0$ and then the spaces $H^{-s}(\partial \Omega)$ may be regarded as dual spaces of $H^{s}(\partial \Omega)$. Most of the rest of this paper describes results based on the following theorem which characterizes the boundary trace properties of the classes of (generalized) harmonic functions on $\Omega$.

Theorem 4.1. The restriction of the trace operator $\Gamma: \mathcal{H}^{s+1 / 2}(\Omega) \mapsto H^{s}(\partial \Omega)$ is an isometric isomorphism for any $s \geq \frac{1}{2}$. The trace operator $\Gamma$ has a continuous linear extension $\Gamma_{s}: \mathcal{H}^{s+1 / 2}(\Omega) \mapsto H^{s}(\partial \Omega)$, which is an isometric isomorphism, for each $s<\frac{1}{2}$.

Proof. First consider the case $s \geq \frac{1}{2}$ and assume $u=\sum_{j=0}^{\infty} c_{j} s_{j} \in \mathcal{H}^{s+1 / 2}(\Omega)$. From (3.1),

$$
\|u\|_{s+1 / 2}^{2}:=\sum_{j=0}^{\infty}\left(1+\delta_{j}\right)^{2 s-1} c_{j}^{2} .
$$

Comparing (4.7) with (4.8), we see that $\|\Gamma u\|_{s, \partial \Omega}^{2}=\|u\|_{s+1 / 2}^{2}$, so $\Gamma$ is an isometric map.

It remains to show the surjectivity of $\Gamma$. Choose $f \in H^{s}(\partial \Omega)$ with the Steklov expansion $\sum_{j=0}^{\infty} a_{j} g_{j}$, and consider

$$
\tilde{u}(x):=\sum_{j=0}^{\infty} a_{j}\left(1+\delta_{j}\right)^{1 / 2} s_{j}(x) .
$$

Then $\Gamma \tilde{u}=f$. Since $f \in \mathcal{H}^{s}(\partial \Omega)$,

$$
\|f\|_{s, \partial \Omega}^{2}=\sum_{j=0}^{\infty} a_{j}^{2}\left(1+\delta_{j}\right)^{2 s}<\infty .
$$

Now

$$
\|\tilde{u}\|_{s+1 / 2}^{2}=\sum_{j=0}^{\infty} a_{j}^{2}\left(1+\delta_{j}\right)^{2 s}=\|f\|_{s, \partial \Omega}^{2}<+\infty
$$

so $\tilde{u} \in \mathcal{H}^{s+1 / 2}(\Omega)$ and thus $\Gamma$ is surjective. 
When $s<\frac{1}{2}$ and $u=\sum_{j=0}^{\infty} c_{j} s_{j} \in \mathcal{H}^{s+1 / 2}(\Omega)$ then $u$ is the limit of Steklov partial sums $u_{K}$ taken in the norm of $\mathcal{H}^{s}(\Omega)$. For each $K \geq 1$ we have, see (4.6),

$$
\begin{aligned}
& \Gamma u_{K}=\sum_{j=0}^{K} \frac{c_{j}}{\sqrt{1+\delta_{j}}} g_{j}(x) \in L^{2}(\partial \Omega, d \sigma), \\
& \left\|\Gamma u_{M}\right\|_{s, \partial \Omega}^{2}=\sum_{j=0}^{K} c_{j}^{2}\left(1+\delta_{j}\right)^{2 s-1} .
\end{aligned}
$$

Moreover, $u \in \mathcal{H}^{s+1 / 2}(\Omega)$ implies that $\left\{\Gamma u_{K} \mid K \geq 1\right\}$ is a Cauchy sequence in $H^{s}(\partial \Omega)$. Hence, there exists a unique $\hat{f} \in H^{s}(\partial \Omega)$ representing the limit of $\Gamma u_{K}$ in the strong topology in $H^{s}(\partial \Omega)$ as $K \rightarrow \infty$. Define the map $\Gamma_{s}$ by the assignment

$$
\Gamma_{s}: u \mapsto \hat{f},
$$

then (4.12) yields the following properties of $\Gamma_{s}$ :

(i) $\Gamma_{s}: \mathcal{H}^{s+1 / 2}(\Omega) \mapsto \mathcal{H}^{s}(\partial \Omega)$,

(ii) $\left\|\Gamma_{s} u\right\|_{s, \partial \Omega}=\|\hat{f}\|_{s, \partial \Omega}=\|u\|_{s+1 / 2}$ in view of (3.1) and (4.12b), and

(iii) $\Gamma_{s}$ is surjective, which is proved just as for the case $s \geq \frac{1}{2}$ above.

It is worth noting that with these definitions when $u \in \mathcal{H}_{F}(\Omega)$ then $\Gamma u \in H^{s}(\partial \Omega)$, for all real $s$ as the partial sums have only finitely many non-zero coefficients in their Steklov expansion.

\section{Generalized Harmonic Dirichlet Problem}

The classical harmonic Dirichlet problem on $\Omega$ is, given a function $f$ on $\partial \Omega$, to find a solution $\hat{u} \in C^{2}(\Omega) \cap C^{0}(\bar{\Omega})$ satisfying

$$
\begin{array}{cc}
-\Delta u(x)=0 & \text { for all } \quad x \in \Omega, \\
\text { subject to } & u(x)=f(x) \quad \text { for all } \quad x \in \partial \Omega .
\end{array}
$$

A necessary, but not sufficient, condition for this to have a classical solution is that $f$ be a continuous function. Our interest is in finding generalized solutions of this problem when $f$ is a Lebesgue integrable function on $\partial \Omega$ that need not be continuous.

The usual weak form of this problem is to find a function $\hat{u} \in \mathcal{H}(\Omega)$ such that

$$
\Gamma \hat{u}=f \quad \text { on } \partial \Omega \text {. }
$$

From the analysis of the previous section, this problem has a unique solution if and only if $f \in H^{1 / 2}(\partial \Omega)$.

When $f \in H^{s}(\partial \Omega)$ for some $s<\frac{1}{2}$ but $f$ is not in $H^{1 / 2}(\partial \Omega)$, then the generalized harmonic Dirichlet problem will be to find $\hat{u} \in \mathcal{H}^{s+1 / 2}(\Omega)$ satisfying

$$
\Gamma_{s} u=f \quad \text { on } \partial \Omega .
$$

Here $\Gamma_{s}$ is the extension of the trace operator to the class $\mathcal{H}^{s+1 / 2}(\Omega)$ of generalized harmonic functions on $\Omega$. This may be regarded as a spectral solution of this problem in 
that the generalized Fourier coefficients of the solution $\hat{u}$ with respect to the orthonormal basis $\mathcal{S}_{1}$ of $L^{2}(\partial \Omega, d \sigma)$ must agree with those of $f$. This follows from (5.3) as $\hat{u}$ satisfies

$$
\left\langle\Gamma_{s} \hat{u}, g_{j}\right\rangle_{\sigma}=\left\langle f, g_{j}\right\rangle_{\sigma} \quad \text { for all } \quad j \geq 0 .
$$

Here the pairing is between $H^{s}(\partial \Omega)$ and $H^{-s}(\partial \Omega)$ via the inner product on $L^{2}(\partial \Omega, d \sigma)$. Theorem 4.1 implies the following result about this problem:

Theorem 5.1. Assume $\Omega$ satisfies (B2), $s \in \mathbb{R}$ and the spaces $\mathcal{H}^{s+1 / 2}(\Omega), H^{s}(\partial \Omega)$ are defined as above. For each $f \in H^{s}(\partial \Omega)$, there is a unique $\hat{u} \in \mathcal{H}^{s+1 / 2}(\Omega)$ which solves (5.3). When $f$ has the Steklov expansion, $f=\sum_{j=0}^{\infty} c_{j} g_{j}$, this solution is

$$
\hat{u}=\sum_{j=0}^{\infty} \sqrt{1+\delta_{j}} c_{j} s_{j}
$$

and the partial sums of this series converge in norm to $\hat{u}$ on $\mathcal{H}^{s+1 / 2}(\Omega)$.

Proof. This is just a restatement of the analysis in Theorem 4.1.

It is useful to detail this explicitly for the situation where the boundary data is $L^{2}$. Assume $f \in L^{2}(\partial \Omega, d \sigma)$ has the Steklov expansion

$$
f(x)=\sum_{j=0}^{\infty}\left\langle f, g_{j}\right\rangle_{\sigma} g_{j}(x) .
$$

It follows from Theorem 4.1 with $s=0$ that there exists unique $\hat{u} \in \mathcal{H}^{1 / 2}(\partial \Omega)$, which satisfies (5.2) and, moreover,

$$
\hat{u}(x)=\lim _{M \rightarrow+\infty} \sum_{j=0}^{M} \sqrt{1+\delta_{j}}\left\langle f, g_{j}\right\rangle_{\sigma} s_{j}(x) .
$$

For any integer $M$, we consider the function $\mathcal{P}_{M}: \bar{\Omega} \times \partial \Omega \mapsto \mathbb{R}$ defined by

$$
\mathcal{P}_{M}(x, y)=\sum_{j=0}^{M} \sqrt{1+\delta_{j}} s_{j}(x) g_{j}(y) .
$$

It follows from the properties of the Steklov eigenfunctions discussed above that $\mathcal{P}_{M}(\cdot, y)$ is harmonic and $C^{\infty}$ on $\Omega$ for each $y \in \partial \Omega$. Multiply this by $f$ and integrate to obtain

$$
\begin{aligned}
\frac{1}{\sigma(\partial \Omega)} \int_{\partial \Omega} \mathcal{P}_{M}(x, y) f(y) d \sigma(y) & \\
= & \sum_{j=0}^{M} \sqrt{1+\delta_{j}}\left\langle f, g_{j}\right\rangle_{\sigma} s_{j}(x) \quad \text { for all } \quad x \in \Omega .
\end{aligned}
$$

Define

$$
E_{M}(f)(x):=\frac{1}{\sigma(\partial \Omega)} \int_{\partial \Omega} \mathcal{P}_{M}(x, y) f(y) d \sigma(y) .
$$


Then $E_{M}(f)$ is a harmonic function that is $C^{\infty}$ on $\Omega$ and Theorem 4.1 guarantees that the unique solution of (5.2) will be such that

$$
E_{M}(f) \rightarrow \hat{u} \quad \text { in } \mathcal{H}^{1 / 2}(\Omega), \text { as } \quad M \rightarrow+\infty .
$$

This provides a sequence of smooth harmonic functions that converge strongly in the $\mathcal{H}^{1 / 2}$-norm to a generalized solution of this harmonic Dirichlet problem that may not be an $H^{1}$-solution. Note that the $\mathcal{H}^{1 / 2}$-norm is stronger than the $L^{2}$ norm on $\Omega$, so the partial sums converge in $L^{2}(\Omega)$. From (5.4), the boundary trace of the functions $E_{M}(f)$ converges strongly to $f$ in $L^{2}(\partial \Omega, d \sigma)$ as $M \rightarrow \infty$.

\section{Steklov Eigenpairs on the Unit Disc}

In this section the preceding analysis is used to obtain formulae for generalized solutions of Dirichlet boundary value problems for Laplace's equation on the unit disc $B(0,1) \subset$ $\mathbb{R}^{2}$. For the unit disc, the Steklov eigenvalues and eigenfunctions are easily determined. $\delta_{0}=0$ is a simple eigenvalue with corresponding eigenfunction $s_{0}=1$. For $k \geq 1$, the Steklov eigenvalues occur in pairs, satisfying

$$
\delta_{2 k-1}=\delta_{2 k}=2 \pi k .
$$

The corresponding normalized eigenfunctions, in polar coordinates, are

$$
\begin{aligned}
& s_{2 k-1}(r, \theta):=a_{k} r^{k} \sin (k \theta), \quad \text { and } \quad s_{2 k}(r, \theta):=a_{k} r^{k} \cos (k \theta), \\
& a_{k}=\sqrt{\frac{2}{2 \pi k+1}} .
\end{aligned}
$$

The coefficient $a_{k}$ here was chosen so that the orthogonality condition (2.7) holds.

The traces of these eigenfunctions are $L^{2}$-orthogonal with respect to arc-length on the boundary $S_{1}=\partial B(0,1)$-which is the unit circle. The associated surface traces defined as in (4.2) are $g_{0}(\theta) \equiv 1$ and

$$
g_{2 k-1}(\theta)=\sqrt{2} \sin (k \theta) \quad \text { and } \quad g_{2 k}(\theta)=\sqrt{2} \cos (k \theta) \quad \text { for } \quad k \geq 1
$$

and these form an $L^{2}$-orthonormal basis on the boundary with the inner product from $(2.2)$.

\subsection{Boundary Data with Singularities}

Consider the function

$$
f(r, \theta)=\log \left(-\log \left(r(1-r)+\theta(2 \pi-\theta) /(2 \pi)^{2}\right)\right) r^{2} \quad \text { on } B(0,1) .
$$

The function $f$ is in $H^{1}(B(0,1))$ but has a singularity at $\theta=0$ or $2 \pi$ and $r=1$. In other words, it is not in $L^{\infty}\left(S_{1}\right)$ but it does belong to $H^{1 / 2}\left(S_{1}\right)$. Since the function is symmetric around $\theta=\pi$, all coefficients in the Steklov expansion corresponding to sinterms are zero. Hence the expansion only involves the cosine terms. The result, shown at Figure 1, exhibits a large number of oscillations near the singularity, corresponding to the Gibbs phenomenon. These oscillations diminish with increasing number of terms in the 


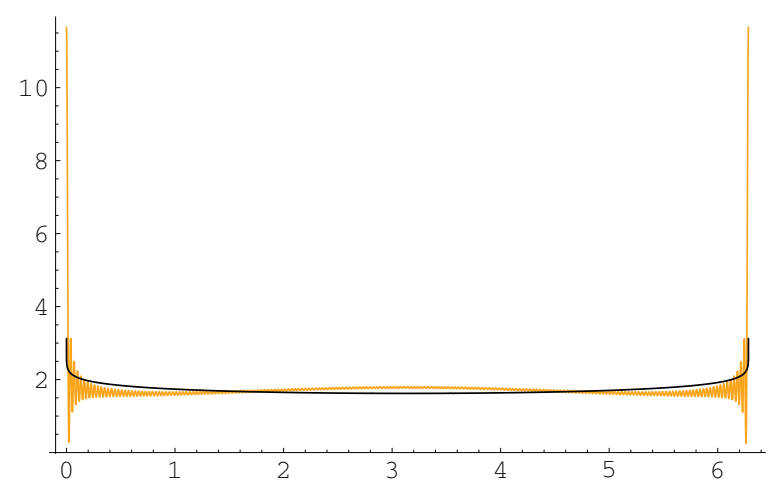

Figure 1. Plot shows the trace $\Gamma f(r, \theta)=\log \left(-\log \left(\theta(2 \pi-\theta) /(2 \pi)^{2}\right)\right)$ and the trace of the Steklov approximation on the boundary of the unit disk. We used 200 terms in the Steklov expansion to obtain this result combined with high precision numerical integration to evaluate the Steklov coefficients.

expansion but never disappear. The oscillations in the Steklov approximation decrease rapidly inside the unit disc.

\subsection{Boundary Data not in $H^{1 / 2}(\partial B(0,1))$}

Consider the problem of finding a generalized solution of Laplace's equation on the unit disc subject to the boundary data

$$
u(1, \theta)=f_{2}(\theta)=(\theta / 2 \pi)^{2},
$$

where $0 \leq \theta \leq 2 \pi$. This function has a finite jump at $(1,0)$ but satisfies $0 \leq f_{2}(\theta) \leq 1$ on $S_{1}$, so it is in $L^{\infty}\left(S_{1}\right)$ and $L^{2}\left(S_{1}\right)$. It is straightforward to show that the Fourier series of this function is

$$
f_{2}(\theta)=\frac{1}{3}+\frac{1}{\pi^{2}} \sum_{k=1}^{\infty} \frac{1}{k^{2}}(\cos k \theta-k \pi \sin k \theta) .
$$

Thus in terms of the basis defined by (6.4), we have

$$
f_{2}(\theta)=\frac{1}{3} g_{0}+\frac{1}{\sqrt{2} \pi^{2}} \sum_{k=1}^{\infty} \frac{1}{k^{2}}\left[g_{2 k}(1, \theta)-k \pi g_{2 k-1}(1, \theta)\right] .
$$

It follows that $f_{2}$ is in $H^{s}(\partial B(0,1))$ for all $s<\frac{1}{2}$ from the definitions of Section 4 and standard convergence results. Thus, from Theorem 5.1, the generalized harmonic function that has $f_{2}$ as its trace on the unit circle is

$$
u(r, \theta):=\frac{1}{3}+\frac{1}{\pi^{2}} \sum_{k=1}^{\infty} \frac{r^{k}}{k^{2}}[\cos (k \theta)-k \pi \sin (k \theta)] .
$$

This function is in the space $\mathcal{H}^{s}(B(0,1))$ for all $s<1$ but it is not weakly harmonic. 
Using the definition (4.4) of the norm on $\mathcal{H}^{s}\left(S_{1}\right)$, define

$$
\begin{aligned}
& \left\|f_{2}\right\|_{s, M, S_{1}}^{2} \\
& \begin{aligned}
=\frac{1}{3}+(2 \pi)^{-4} \sum_{k=1}^{M}(1+2 \pi k)^{2 s+1} & \left(\left(\frac{1}{2 \pi} \int_{0}^{2 \pi} \theta^{2} \sqrt{2} \sin (k \theta) d \theta\right)^{2}\right. \\
& \left.+\left(\frac{1}{2 \pi} \int_{0}^{2 \pi} \theta^{2} \sqrt{2} \cos (k \theta) d \theta\right)^{2}\right) \\
=\frac{1}{3}+\sum_{k=1}^{M}(1+2 \pi k)^{2 s+1}\left(\left(\frac{1}{\sqrt{2} \pi k}\right)^{2}\right. & \left.+\left(\frac{1}{\sqrt{2} \pi^{2} k^{2}}\right)^{2}\right) .
\end{aligned}
\end{aligned}
$$

Take $M=10^{3}$ in (6.8) then

$$
\left|\left\|f_{2}\right\|_{0,1000, S_{1}}^{2}-\left\|f_{2}\right\|_{L^{2}\left(S_{1}\right)}^{2}\right|<5.1 \times 10^{-5} .
$$

We note that the above inequality is the only way to examine the correctness and the truncation error of the representation formula (6.8) if the boundary datum is not in $H^{1 / 2}\left(S_{1}\right)$; see [7] for details.

\section{Dewetting of a Thin Film}

In order to provide some intuitive understanding of the generalized harmonic function solving a Dirichlet problem with boundary data not in $H^{1 / 2}(\Omega)$ presented in Section 6.2, we consider an idealized thin (e.g., liquid) film attached to a wire frame that is broken at some point. We begin by admitting that, in principle, the generalized minimal surface problem cannot model the rupture on the micro-scale as it is missing two fundamental ingredients: the chemical potential and surface energies. The rupture occurs in the presence of the disjoining pressure, and its kinetics depend strongly on contribution from the membrane density [3]. From the energetics point of view, the process of rupture occurs when the kinetic energy of the oscillating membrane (due to braking the frame) exceeds the surface and elastic energies of the system. The surface (interfacial) energies correspond to liquid-air and liquid-metal-air interfaces. Microscopically, the surface energies are due to the molecular mixture of different chemical compounds at points of their contact. Close to the wire frame, the surface energy of the liquid-metal interface must be higher than the energy needed to separate the molecules of the thin liquid film, for otherwise the membrane would not exist. In other words, there exists a layer, very close to the wire frame, with physical properties, such as elastic modulus, very different from that of liquid film. Mathematical description of the layer and its role has been established by Klouček and Romerio in [8].

The framework presented in this paper allows us to define a "generalized" solution of a Dirichlet problem with boundary datum having a boundary discontinuity, using Theorem 5.1. From a physics point of view, we are modeling a "minimal surface" at the infinitesimal moment after a rupture of the wire frame. In other words, interpreting the 

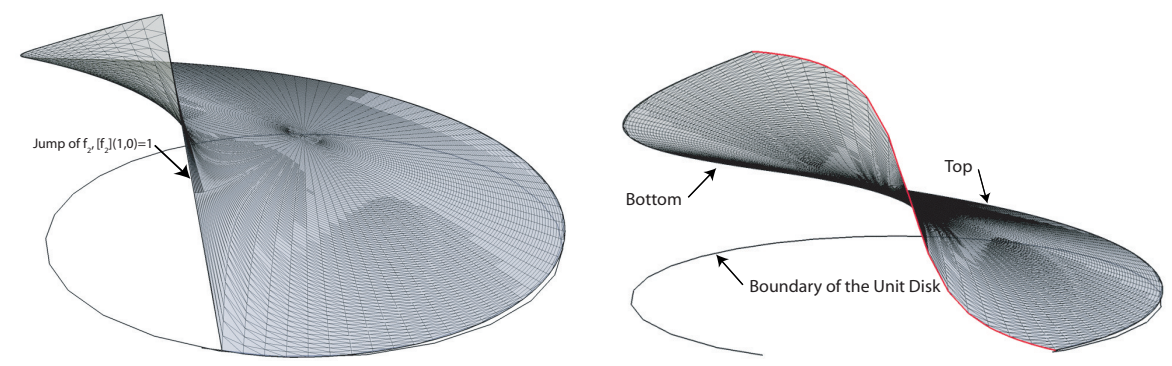

Figure 2. Three-dimensional plot of the generalized harmonic function solving the Dirichlet problem (5.1) with the boundary data $f_{2}$. The slope of the jump, visible on the drawing of the entire Steklov solution in the left picture, is due to the number of terms used in the partial Steklov sum. The picture on the right shows a slice through the surface of the Steklov solution to visualize the curvature of the surface.

"generalized" solution as a deformation, the deformation gradient, which is proportional to internal forces, can be used to find a path of propagating rupture, for it is going to happen at the material points of highest stress.

A three-dimensional drawing of the generalized solution of the Dirichlet problem (5.1) is shown in Figure 2. This figure also shows a slice through the surface of the solution to visualize its curvature. Notice that the Steklov solution satisfies the maximum/minimium principle, i.e.,

$$
\max _{x \in B(0,1)} E_{M}\left(f_{2}\right)(x)=\max _{y \in S_{1}} \mathcal{T}_{M} f_{2}(y) \text { and } \min _{x \in B(0,1)} E_{M}\left(f_{2}\right)(x)=\min _{y \in S_{1}} \mathcal{T}_{M} f_{2}(y),
$$

where $\mathcal{T}_{M}$ indicates the $M$ th partial sum of the Steklov expansion of a function on the boundary.

\subsection{Comparison of the Steklov and Finite Element Solutions}

We illuminate some of the more important differences between the Steklov spectral solution of the Dirichlet problem (5.1) and its Finite element solution. We have mentioned already that this boundary value problem has a weak solution if and only if the boundary data is in $H^{1 / 2}\left(S_{1}\right)$. This precludes finite jumps on the boundary, so for such data, even if we can find a Finite element solution for any $h>0$ we must observe

$$
\left\|u_{h}\right\|_{H^{1}(B(0,1))} \rightarrow+\infty \quad \text { as } \quad h \rightarrow 0 .
$$

Thus no conclusions can be drawn from the discrete Finite element solution of (5.1). The differences between the Steklov and the Finite element solutions are visualized in Figures 3 and 4. Those figures show quite a substantial difference between the two surfaces. The Finite element solution exhibits an excessive amount of curvature visible in Figure 4. This is most likely responsible for the blow-up of the $L^{2}$-norm of the gradients in the above limit pass.

On the other hand, the Steklov solution that minimizes the $\|\cdot\|_{s_{0}+1 / 2}$-norm seems to provide a solution with quite small curvature except near the boundary discontinuity.

We used a polygonal domain with 128 boundary nodes to approximate the Unit Disk for the Finite element computations. We used bilinear, $Q_{1}$-Finite elements. The dimension of the Finite element space used is 5185. 

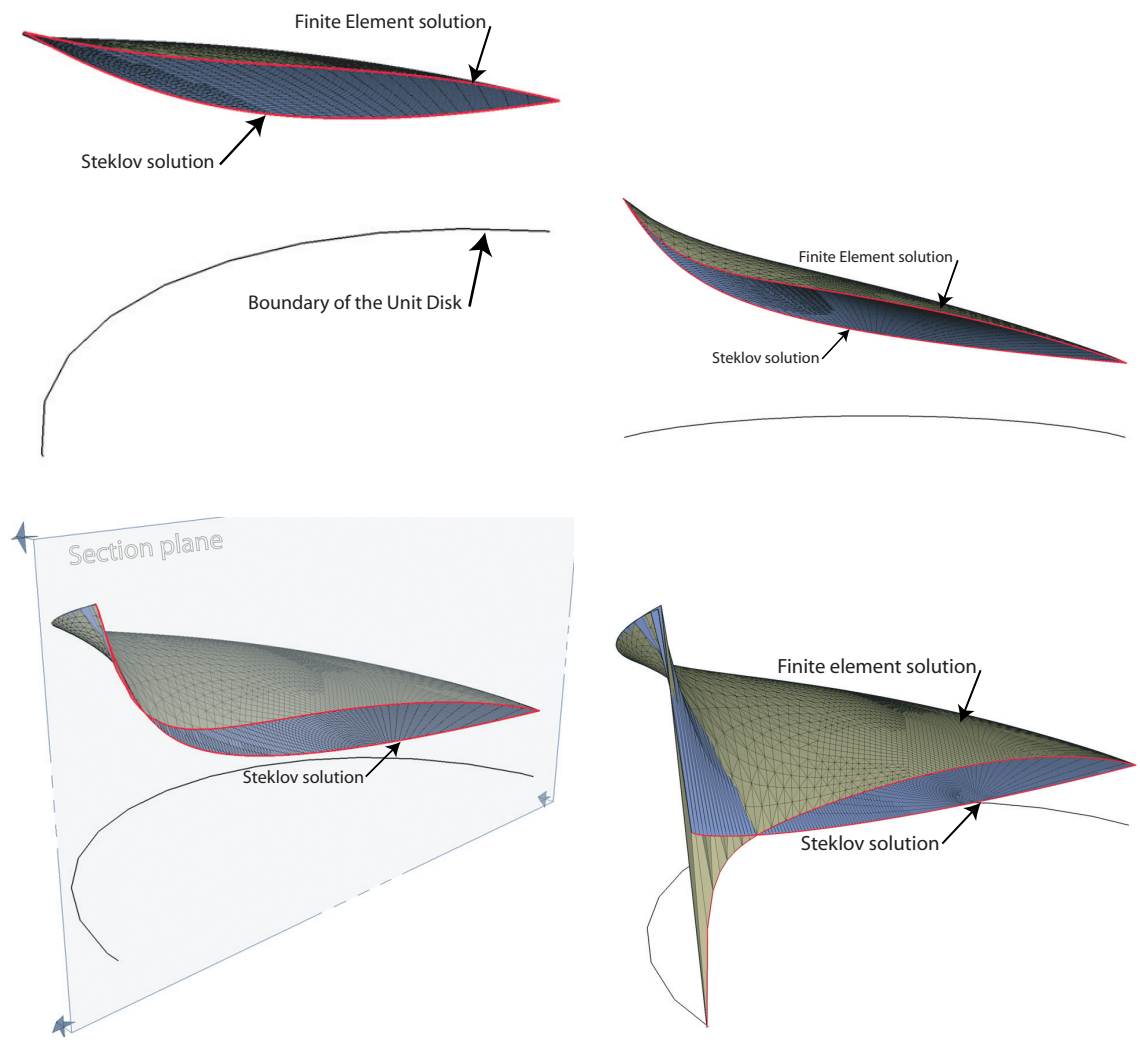

Figure 3. Cross sections through the surfaces corresponding to the Finite element and the Steklov "solution" of the Dirichlet problem (5.1) with the boundary data $f_{2}$. The upper surface (lighter color) is generated using finite elements and the lower surface (darker color) corresponds to the Steklov solution. Notice how different the respective solutions are near the discontinuity on the boundary. The lower-left picture shows the section plane used to obtain the cross sections of the superimposed Steklov and Finite element solutions. In all the cases the section planes are parallel to each other.
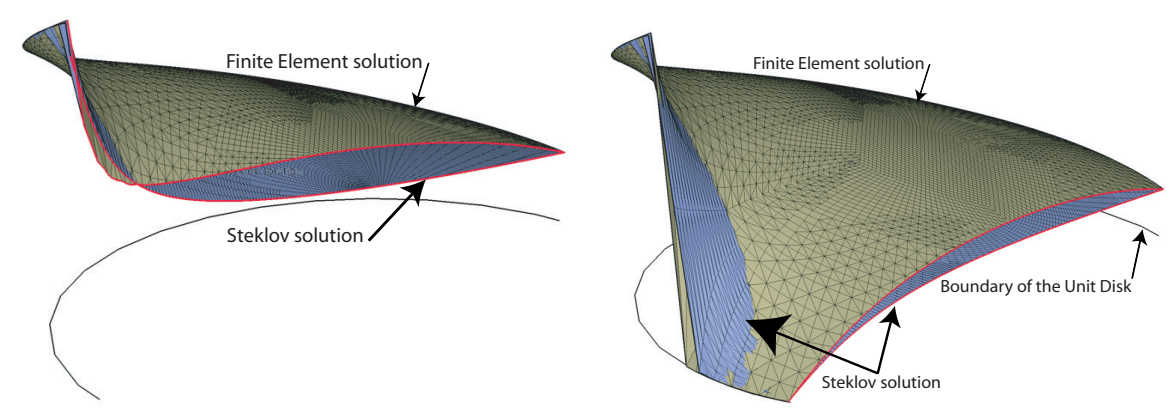

Figure 4. Cross sections through the surfaces corresponding to the Finite element and the Steklov "solution" of the Dirichlet problem (5.1) with the boundary data $f_{2}$. Notice a profound difference in the curvature of the two solutions. As in Figure 3, the lighter color corresponds to the Finite element solution and the darker color corresponds to the Steklov solution. 
The Steklov solution is approximated by a partial sum $E_{500}\left(f_{2}\right)$. All the boundary integrals appearing in the Steklov formula are computed exactly.

\section{Appendix}

This section provides a summary of the definitions used in the paper as well as some important relations.

Lemma A.1. We assume that $\Omega$ and $\partial \Omega$ satisfy (B2). Let $\mathcal{H}^{s}(\Omega)$ be given by Definition 3.1, and let $H^{s}(\partial \Omega)$ be the closure of $L^{2}(\partial \Omega, d \sigma)$ with regard to the norm $\|\cdot\|_{s, \partial \Omega}$. We have

$$
\mathcal{H}^{-s}(\Omega)=\mathcal{H}^{s}(\Omega)^{*} \quad \text { and } \quad H^{-s}(\partial \Omega)=H^{s}(\partial \Omega)^{*} \quad \text { for any } s \in \mathbb{R}
$$

with the duality pairings provided by the inner products $\langle\cdot, \cdot\rangle_{1}$ and $\langle\cdot, \cdot\rangle_{\sigma}$, respectively. (See Table 2 for their respective definitions.) Consequently, both spaces of generalized harmonic functions and the associated trace spaces are reflexive for any $s \in \mathbb{R}$.

Proof. Let $f \in \mathcal{H}^{s}(\Omega), s \geq 0$, and let

$$
\left\langle\mathcal{F}_{f}, v\right\rangle_{0}:=\langle f, v\rangle_{0} \quad \text { for all } \quad v \in \mathcal{H}^{s}(\Omega) .
$$

It follows from the Schwarz inequality applied to the duality $\langle\cdot, \cdot\rangle_{0}$ that $\mathcal{F}_{f} \in \mathcal{H}^{s}(\Omega)^{*}$ for $\mathcal{H}^{s}(\Omega) \subseteq \mathcal{H}^{0}(\Omega)$ if $s \geq 0$; see Theorem 3.2.

Let $\mathcal{F} \in \mathcal{H}^{s}(\Omega)^{*} . \mathcal{H}^{s}(\Omega)$ are real Hilbert spaces with the inner products given by (3.2). The Riesz Representation Theorem [4], [10] asserts that each Hilbert space is isometrically isomorphic to its dual. Hence, there exists $g \in \mathcal{H}^{s}(\Omega)$ such that

$$
\langle\mathcal{F}, v\rangle_{0}:=\langle g, v\rangle_{0} \quad \text { for all } \quad v \in \mathcal{H}^{s}(\Omega) .
$$

Let

$$
g_{j}:=\left[g, s_{j}\right]_{\partial} \quad \text { and } \quad v_{j}:=\left[v, s_{j}\right]_{\partial} .
$$

Then, using the Hölder inequality,

$$
\left|\langle g, v\rangle_{0}\right|=\left|\sum_{j=0}^{\infty} g_{j} v_{j}\right|=\left|\sum_{j=0}^{\infty}\left(1+\delta_{j}\right)^{-(s+1)} g_{j}\left(1+\delta_{j}\right)^{s+1} v_{j}\right| \leq\|g\|_{-s}\|v\|_{s} .
$$

Hence $\mathcal{F}$ is bounded on $\mathcal{H}^{s}(\Omega)$ with equality in (A.2) if $g \in \mathcal{H}^{-s}(\Omega)$ has the expansion coefficients given by $\left[v, s_{j}\right]_{\partial}, j \geq 0$. Since boundedness is synonymous with continuity in the case of linear functionals, we conclude that $\mathcal{F} \in \mathcal{H}^{-s}(\Omega)$ for any $s \geq 0$. 
Table 1. Descriptions of the function spaces used in the paper. The inner products are defined in Table 2 .

\begin{tabular}{llc}
\hline \multicolumn{1}{c}{ Notation } & \multicolumn{1}{c}{ Definition } & \multicolumn{1}{c}{ Remark } \\
\hline$L^{2}(\partial \Omega, d \sigma)$ & Section 2 & $L^{2}(\partial \Omega, d \sigma)=\mathcal{H}^{0}(\partial \Omega)$ w.r.t. $\left\{g_{j}\right\}$ \\
$H^{1}(\Omega)$ & Section 2.1 and [6] & $W^{1,2}(\Omega)$ endowed with an alternative inner \\
& product \\
$\mathcal{H}_{F}(\Omega)$ & Finite linear combinations of $s_{j}$ & $s_{j}$ are Steklov eigenfunctions such that \\
& & {$\left[s_{j}, s_{k}\right]_{\partial}=\delta_{j k}$} \\
$\mathcal{H}(\Omega)$ & Section 2 & $\mathcal{H}(\Omega)=\mathcal{H}^{1}(\Omega)$ and finite energy harmonic \\
& functions \\
$\mathcal{H}^{s}(\Omega)$ & Closure of $\mathcal{H}_{F}(\Omega)$ w.r.t. $\|\cdot\|_{s}$ & If $s<0$ then elements of $\mathcal{H}^{s}(\partial \Omega)$ are \\
$H^{s}(\partial \Omega)$ & Closure of $L^{2}(\partial \Omega, d \sigma)$ w.r.t. $\|\cdot\|_{s, \partial \Omega}^{2}$ & generalized functions \\
& &
\end{tabular}

Assuming existence of two elements $g_{1}, g_{2} \in \mathcal{H}^{s}(\Omega)$ representing $\mathcal{F}$ we get

$$
\left\langle g_{1}-g_{2}, s_{j}\right\rangle_{s}=0, \quad j=0,1,2, \ldots,
$$

where $s_{j} \in \mathcal{H}^{s}(\Omega)$ are Steklov eigenfunctions forming a basis in $\mathcal{H}^{s}(\Omega)$. Hence $g_{1}=g_{2}$ in $\mathcal{H}^{s}(\Omega)$.

The duality for the trace spaces follows from Theorem 4.1. Namely, according to this theorem, $\mathcal{H}^{s+1 / 2}(\Omega)$ and $H^{s}(\partial \Omega)$ are isometrically isomorphic when $H^{s}(\partial \Omega)$ is an image of $\mathcal{H}^{s+1 / 2}(\Omega)$ under the trace operator

$$
T:=\left\{\begin{array}{lll}
\Gamma, & s \geq \frac{1}{2}, & \Gamma: H^{1}(\Omega) \mapsto L^{2}(\partial \Omega, d \sigma) \text { compact } \\
& \text { i.e., assumption (B2) }, \\
\text { cont. lin. ext. of } \Gamma_{s} & s<\frac{1}{2}, & \Gamma_{s} \text { given by (4.13). }
\end{array}\right.
$$

Thus the duality result for the generalized trace spaces follows from the first part of the theorem.

Table 2. Dualities and inner products used in the paper. The function $s_{j}$ is a Steklov eigenfunction corresponding to the $j$ th eigenvalue of (2.6) and normalized as in (2.7). The $g_{j}$ is the boundary trace of this eigenfunction defined by $g_{j}(x)=\sqrt{1+\delta_{j}} \Gamma s_{j}(x), x \in \partial \Omega$.

\begin{tabular}{lc}
\hline \multicolumn{1}{c}{ Space } & \multicolumn{1}{c}{ Inner product } \\
\hline$L^{2}(\partial \Omega, d \sigma)$ & $\langle u, v\rangle_{\sigma}:=\sigma(\partial \Omega)^{-1} \int_{\partial \Omega} u v d \sigma$ \\
$H^{1}(\Omega)$ & {$[u, v]_{\partial}:=\int_{\Omega} \nabla u(x) \cdot \nabla v(x) d x+\langle\Gamma u, \Gamma v\rangle_{\sigma}$} \\
$\mathcal{H}(\Omega)$ & $\langle u, v\rangle_{1}:=\sum_{j=0}^{\infty}\left[u, s_{j}\right]_{\partial}\left[v, s_{j}\right]_{\partial}$ \\
$\mathcal{H}^{s}(\Omega)$ & $\langle u, v\rangle_{s}:=\sum_{j=0}^{\infty}\left(1+\delta_{j}\right)^{2(s-1)}\left[u, s_{j}\right]_{\partial}\left[v, s_{j}\right]_{\partial}$ \\
$H^{s}(\partial \Omega)$ & $\langle\Gamma u, \Gamma v\rangle_{s, \partial \Omega}:=\sum_{j=0}^{\infty}\left(1+\delta_{j}\right)^{2 s+1}\left\langle\Gamma u, \Gamma s_{j}\right\rangle_{\sigma}\left\langle\Gamma v, \Gamma s_{j}\right\rangle_{\sigma}$ \\
\hline
\end{tabular}




\section{References}

1. G. Auchmuty, Steklov Eigenproblems and representation of solutions of elliptic boundary value problems, Numer. Func. Anal. Optim. 25(3-4) (2004), 321-348.

2. G. Auchmuty, Spectral characterization of the trace spaces $H^{s}(\partial \Omega)$, SIAM J. Math. Anal. (2005), to appear.

3. V. Bergeron, Forces and structures in thin liquid soap films, J. Phys. Condens. Matter 11 (1999), 215-238.

4. L. C. Evans, Partial Differential Equations, Graduate Studies in Mathematics, vol. 19, American Mathematical Society, Providence, RI, 1998.

5. L. C. Evans and R. F. Gariepy, Measure Theory and Fine Properties of Functions, CRC Press, Boca Raton, FL, 1992.

6. S. Fučík, O. John, and A. Kufner, Function Spaces, Academia, Prague, 1977.

7. P. Klouček, D. Sorensen, and J. Wightman, The approximation and computation of a basis of the trace space $H^{1 / 2}$, J. Sci. Comput. (2006), to appear.

8. P. Klouček and M. V. Romerio, The detachment of bubbles under a porous rigid surface during aluminium electrolysis, Math. Models Methods Appl. Sci. 12(11) (2002), 1617-1652.

9. E. Magenes and J. L. Lions, Problèmes aux limites non homogènes et applications, Dunod, Paris, 1968.

10. K. Yosida, Functional Analysis, Springer-Verlag, Berlin, 1971.

11. E. Zeidler, Nonlinear Functional Analysis and Its Applications, Vol. 2/A, Springer-Verlag, New York, 1990.

Accepted 9 July 2006. Online publication 7 March 2007. 